\title{
CMOS-compatible silicon nitride spectrometers for lab-on-a-chip spectral sensing
}

\author{
Eva Ryckeboer ${ }^{\mathrm{a}}$, Xiaomin Nie ${ }^{\mathrm{a}}$, Ananth Z. Subramanian ${ }^{\mathrm{a}}$, Daan Martens ${ }^{\mathrm{a}}$, Peter Bienstman ${ }^{\mathrm{a}}$, \\ Simone Severi ${ }^{\mathrm{b}}$, Roelof Jansen ${ }^{\mathrm{b}}$, Gunther Roelkens ${ }^{\mathrm{a}}$, and Roel Baets ${ }^{\mathrm{a}}$

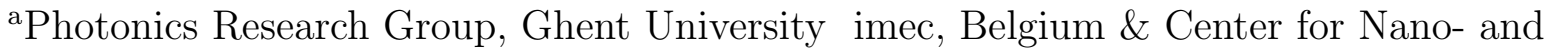 \\ Biophotonics, Ghent University, Belgium \\ bimec, Kapeldreef 75, Heverlee, Belgium
}

\begin{abstract}
We report on miniaturized optical spectrometers integrated on a photonic integrated circuit (PIC) platform based on silicon nitride waveguides and fabricated in a CMOS-compatible approach. As compared to a siliconon-insulator PIC-platform, the usage of silicon nitride allows for operation in the visible and near infrared. Furthermore, the moderately high refractive index contrast in silicon-nitride photonic wire waveguides provides a valuable compromise between compactness, optical loss and sensitivity to phase error. Three generic types of onchip spectrometer are discussed: the arrayed waveguide grating (AWG) spectrometer, the echelle grating or planar concave grating (PCG) spectrometer and the stationary Fourier transform spectrometer (FTS) spectrometer. Both the design as well as experimental results are presented and discussed. For the FTS spectrometer a specific design is described in detail leading to an ultra-small $\left(0.1 \mathrm{~mm}^{2}\right)$ footprint device with a resolution of $1 \mathrm{~nm}$ and a spectral range of 100nm. Examples are given of the usage of these spectrometers in refractive index biosensing absorption spectroscopy and Raman spectroscopy.
\end{abstract}

Keywords: Integrated photonics, miniature spectrometers, absorption spectroscopy, Raman spectroscopy, silicon, silicon-nitride

\section{INTRODUCTION}

Optical spectroscopy is an important method to analyze the composition of a sample. It has the advantage of high specificity because it relies on the unique and "fingerprint" nature of the sample's spectrum. In the past decade, photonic integration technologies made it possible to develop a complete on-chip spectrometer that is both low-cost, portable and robust. At the same time, on-chip spectrometers can have performance specifications that exceed their bulk optic counterparts in terms of resolution and multiplexing capabilities. This has been shown by various miniature spectrometer demonstrators in the Si photonics, Triplex or Indium Phosphide (InP) photonic integration platforms. ${ }^{1-3}$

Si photonics technology comprises the silicon-on-insulator (SOI) and silicon nitride $\left(\mathrm{Si}_{3} \mathrm{~N}_{4}\right)$ material platforms and is intensively studied as it allows for cost-effective mass fabrication in a CMOS fab. This results from the high-yield and nm-accurate processing of complex optical circuits including passive optical components, thermooptic phase-tuners, optical modulators and detectors on wafers up to $300 \mathrm{~mm}$ in diameter. For the SOI material platform, the operational wavelength range is limited to $1.1 \mathrm{um}-3.5 \mathrm{um}$. For shorter wavelengths, the silicon waveguide layer starts to absorb and the upper wavelength limit marks the onset of opacity of the silicon oxide cladding. Therefore, for wavelengths below $1.1 \mathrm{um}$, silicon can be used as a detector material and silicon nitride as the waveguide layer. This is the basis for the $S i_{3} N_{4}$ material platform. This technology platform has excellent optical properties such as low loss and low sensitivity to phase noise. This is beneficial for integrated spectrometer designs as it reduces both the insertion loss and crosstalk level of any wavelength demultiplexer. Moreover, the $\mathrm{Si}_{3} N_{4}$ waveguides can readily be used for efficient and field-enhanced evanescent sensing, giving way to performant on-chip absorption, fluorescence and Raman spectrometers.

Further author information: (Send correspondence to Eva Ryckeboer.)

Eva Ryckeboer: E-mail: eva.ryckeboer@ugent.be, Telephone: 003292649959 
Different implementations of on-chip spectrometers exist. Two common types are the arrayed waveguide grating (AWG) and echelle grating or planar concave grating (PCG). The former is the workhorse of wavelength (de)multiplexing in data/telecom applications and the latter is the planar implementation of a classic diffraction grating spectrometer. Next to these dispersive spectrometers, also Fourier transform spectrometers (FTS) have been integrated on chip. These can offer high resolution over a large wavelength range. Still, many research efforts are targeted to decrease the size of the complete FTS for a given resolution. In this work, we will discuss how a novel FTS design can achieve this challenging combination of small size $\left(1 \mathrm{~mm}^{2}\right)$, high resolution $(1 \mathrm{~nm})$ and large bandwidth $(100 \mathrm{~nm})$.

The outline of this paper is as follows: We will start by explaining the advantages of the $S i_{3} N_{4}$ material platform for on-chip spectrometers. Secondly, we report on the design and measurement results of a $\mathrm{Si}_{3} \mathrm{~N}_{4}$-based AWG and PCG spectrometer that were designed for two distinct life science applicatons. Finally, we discuss a novel FTS design in detail that is suitable for on-chip Raman spectroscopy.

\section{SILICON NITRIDE MATERIAL PLATFORM FOR ON-CHIP SPECTROMETERS}

Silicon nitride is a promising platform for on-chip spectrometers as it shares the mature CMOS process technology with silicon, while offering more tolerant photonic integrated chip designs and a wider operational bandwidth. This can be seen by taking a look at the optical and material properties of $S i_{3} N_{4}$ which we shortly discuss here. Silicon nitride is a dielectric material that is transparent from 0.4-5 um and can be deposited on silicon oxide by low-pressure (LP-CVD) or plasma enhanced (PE-CVD) chemical vapor deposition. Depending on the deposition technique and settings the refractive index $n$ of the $S i_{3} N_{4}$ is different. ${ }^{4}$ Typical curves for the refractive index of both LP-CVD and PE-CVD are shown in figure 1. Compared to silicon waveguides, $S i_{3} N_{4}$ waveguides have a

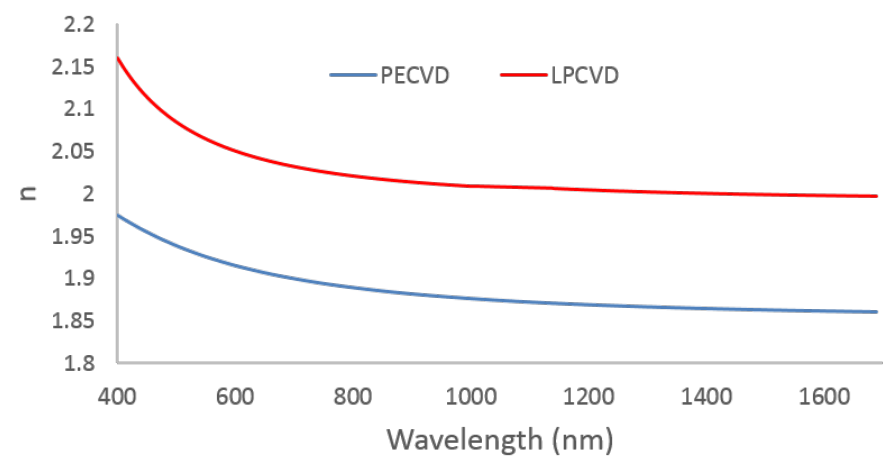

Figure 1: Typical values of the refractive index in function of wavelength of bulk $S i_{3} N_{4}$ deposited using PECVD (blue) and LPCVD (red).

lower index contrast between the waveguide and cladding layer. This results in a waveguide effective index that varies relatively little with the small dimensional variations due to fabrication along the waveguide. This lowers the phase noise and losses due to distributed scattering. Eventually these two positive effects allow to create better on-chip spectrometers. To explain this, we focus on 3 different requirements for on-chip spectrometers. Firstly, the insertion loss should be low to enable power-efficient devices. A low insertion loss can be obtained in the $S i_{3} N_{4}$ material platform because the lower index contrast reduces the waveguide losses caused by sidewall roughness. In addition, $\mathrm{Si}_{3} N_{4}$ doesn't suffer from two-photon absorption. Therefore the nonlinear losses are also low, even when injecting high power intensities. For ridge waveguides, low losses of $<1 \mathrm{~dB} / \mathrm{cm}$ have been regularly achieved. ${ }^{5}$ These low losses allow to increase the optimal interaction length for evanescent sensing which enhances spectrometer sensitivity. ${ }^{6,7}$ To increase the interaction length while keeping a small footprint, the sensing waveguides can be routed into a spiral-shape. In a $S i_{3} N_{4}$ spiral waveguide, the bends can have losses as low as $0.015 \mathrm{~dB} / 90$ degree bend for a radius of 20 micron. ${ }^{8}$ This small radius allows for compact spectrometer circuits. 
A second requirement for spectrometers is low spectral channel crosstalk. Due to crosstalk, the background signal from out-of-channel wavelengths is increased, thereby lowering the signal-to-noise ratio (SNR) of the spectrometer. Because of the reduced index contrast in $S i_{3} N_{4}$ waveguides and the accompanying lower phase noise, a more accurate splitting of the different wavelengths in a demultiplexer can be achieved. This leads then to a spectrometer with lower channel crosstalk and a better SNR can be realized.

A final requirement for spectrometers is spectral stability with respect to environmental parameters such as pressure, temperature, humidity etc. The environmental parameters can impact the waveguides in two ways. Either the effective index changes or the absorption changes. The latter effect is an additional insertion loss, but the former can lead to an unwanted shift in the spectral response of the spectrometer. Moreover, spurious reflections that originate from the index mismatch of abruptly varying waveguide dimensions, are subject to these changes in the effective index. These spurious reflections create a fine spectral ripple in the optical spectrum which is considered spectral noise because of the complex dependency on the environmental parameters. As $\mathrm{Si}_{3} \mathrm{~N}_{4}$ waveguides have a reduced index contrast, the magnitude of the spurious reflections and ripple will decrease, leaving us with an improved spectrometer. Also the thermal material properties of $S i_{3} N_{4}$ help in achieving a thermally stable spectrometer. Compared to silicon, $S i_{3} N_{4}$ has a 10 times lower thermo-optic coefficient $\frac{\delta n}{\delta t}=4.0 \cdot 10^{-5} \frac{R I U}{K} \cdot{ }^{9}$ This will lower the spectral shift that is generated by a variation in temperature and the above-described ripple. It can be concluded that the $S i_{3} N_{4}$ material platform offers the right balance between compactness, optical loss and sensitivity to phase errors.

\section{ARRAYED WAVEGUIDE GRATING SPECTROMETER}

Arrayed waveguide gratings (AWGs) are mainly applied in optical communications but are increasingly being used in a range of lab-on-a-chip based photonic sensing systems, ranging from spectroscopy to refractive index sensing. The shift to the latter has spurred interest in designs in numerous wavelength ranges, diverting from the telecom range. ${ }^{10}$ The very near-infrared range is of particular interest due to its negligible water absorption and low-cost sources and detectors. Here we describe the use of an AWG implemented in $\mathrm{Si}_{3} \mathrm{~N}_{4}$ for tuberculosis antigen detection in urine that operates in the very near-infrared. ${ }^{11}$

AWGs are feed-forward spectral filters, where a series of delay lines connects two free-space focusing regions, referred to as star couplers, as illustrated in part a of Fig. 2. The fixed length difference between the delay lines results in different sections of the spectrum interfering constructively in different locations in the image plane. ${ }^{12}$
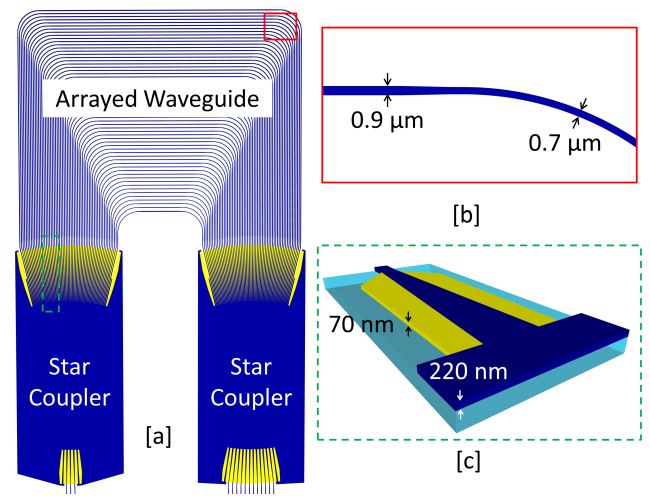

[b]
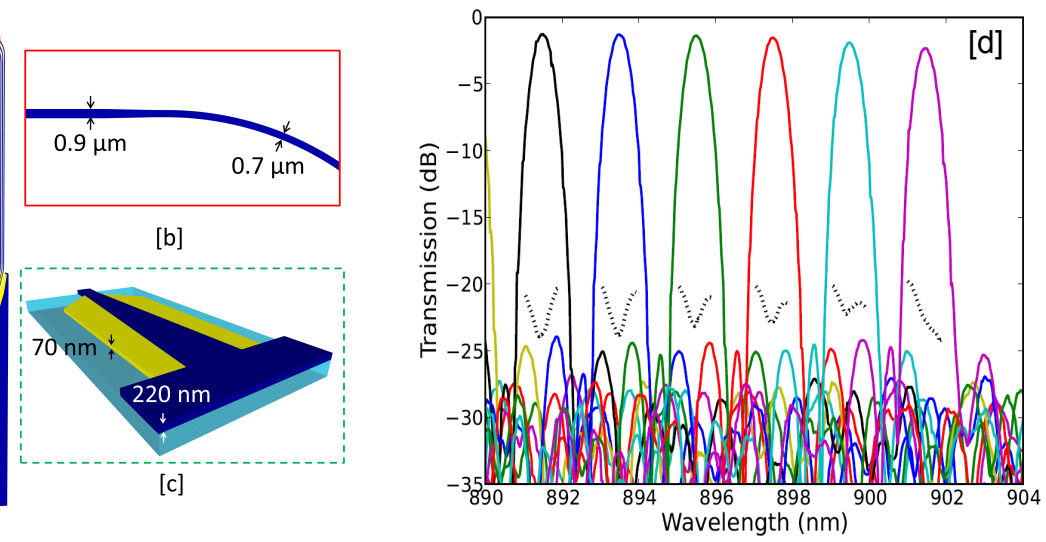

Figure 2: (a) Top view of the arrayed waveguide grating design (b) Close-up on the arrayed waveguide section (c) Close up on the access waveguide (d) Measured spectral response of the AWG. The grey dotted lines show the cumulative crosstalk.

As explained before, $S i_{3} N_{4}$ is a moderately high-index-contrast platform so that the footprint of these devices can be quite low due to the availability of sharp bends. In this case the total AWG size is $0.34 \mathrm{~mm}^{2}$. To enhance 
the AWG performance in terms of insertion loss and spurious reflections, the following two main adaptations were made. Firstly, the most critical location in terms of reflections and insertion loss is the transition from the input and output waveguides to the free-space propagation region (part c of Fig. 2). Here an adiabatic transition is implemented by applying a double etch step in combination with a widening waveguide. Secondly, in the straight sections of the arrayed waveguides, the waveguides are widened from 0.7 to $0.9 \mu \mathrm{m}$ to reduce the influence of the sidewalls, as illustrated in part b of Fig. 2. The AWG design was realized using $193 \mathrm{~nm}$ optical lithography on a Si wafer with a $2.6 \mu \mathrm{m}$ box and $220 \mathrm{~nm}$ thick $S i_{3} N_{4}$ layer. Multiple devices were fabricated, for example a 12-channel AWG centered around $890 \mathrm{~nm}$, with $2 \mathrm{~nm}$ channel spacing. A measurement result of this 12-channel AWG is given in 2(d). Here only 6 out of 12 channels are shown because of the limited tunability of the laser source that was used for characterization. We measured an insertion loss of only $1.2 \mathrm{~dB}$, combined with a reasonable crosstalk value of $20 \mathrm{~dB}$, indicating successful reduction of losses due to sidewall scattering. Other devices with a lower channel spacing of $1 \mathrm{~nm}$ but with 20 and 30 spectral channels, yielded a similar low insertion loss, albeit with a slightly higher crosstalk. ${ }^{13}$ This is due to the increase in AWG size for a smaller channel spacing which leads to an accumulation of phase errors.

These AWG devices can enable cost-effective refractive index sensors for point-of-care applications. Whereas typically a tunable laser is used to interrogate the wavelength-shift due to a refractive index change of the sensor, here a broadband source suffices. In such a scheme (see Fig. 3a), we employ a Mach-Zehnder interferometer (MZI) sensor in combination with a 30-channel AWG with $1 \mathrm{~nm}$ channel spacing. The full sensor spectrum is sampled by the AWG, and by curve fitting, the original spectrum is reconstructed, resulting in a precise localization of the peak wavelengths. This way, a minimal detectable wavelength shift of $4 \mathrm{pm}$ and a sensitivity of $5440 \mathrm{~nm} / \mathrm{RIU}$ was realized, resulting in a limit of detection of $7 \cdot 10^{-7}$ RIU. More details on the set-up and sensitivity measurements can be found in. ${ }^{14}$ The sensorgram of a refractive index sensing experiment with this point-of-care device is shown in Fig. 3b. It shows the extracted refractive index change due to different phosphate buffered saline (PBS) solutions that are flown across the MZI sensing arm. As the free spectral range of the MZI is $7 \mathrm{~nm}$ and the AWG can monitor over a range of $30 \mathrm{~nm}$, four different interference peaks are detected simultaneously. The wavelength shift of all these peaks clearly correlate with the change in PBS concentration. This interrogator scheme has a flat noise floor contributing to the low limit of detection which is comparable to state-of-the-art refractive index sensors. ${ }^{15}$ This enables this sensor to be be used for tuberculosis antigen detection in urine.

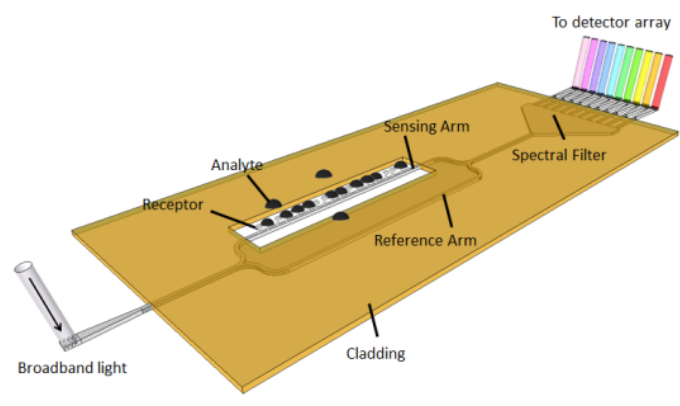

(a)

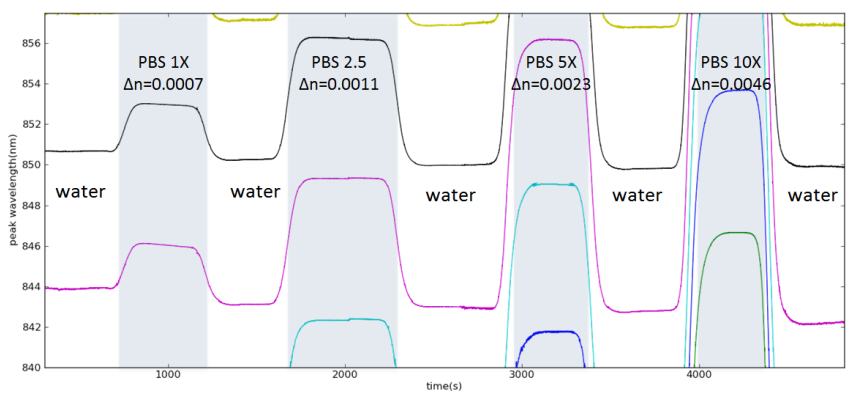

(b)

Figure 3: (a) Illustration of a miniature refractive index sensor combining an MZI interferometer with the AWG on-chip spectral filter (b) Sensorgram achieved in a characterization experiment. Multiple variations of PBS are flown onto the sample, alternated with water.

\section{A LOW CROSSTALK PLANAR CONCAVE GRATING SPECTROMETER}

In this section, we discuss a planar concave grating (PCG) which is a second type of dispersive spectrometer. A PCG, also known as echelle grating, is the planar version of the classic diffraction grating spectrometer. Light from an input waveguide enters a free propagating region (slab waveguide) where it spreads and hits onto a 


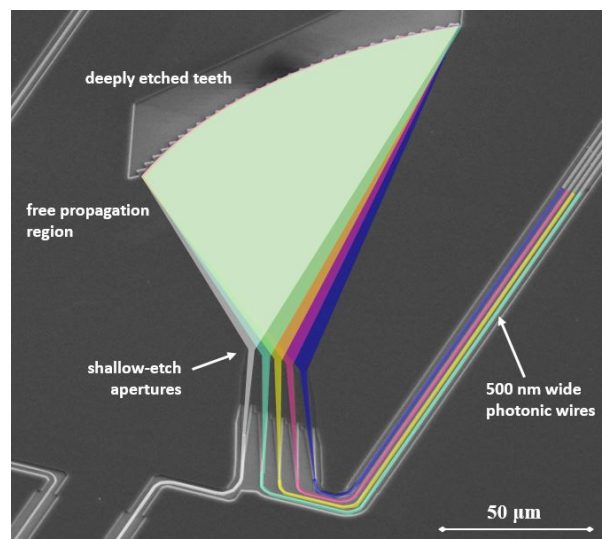

Figure 4: Illustration of the working principle of a planar concave grating spectrometer ${ }^{16}$

curved diffraction grating and gets simultaneously reflected and diffracted by the grating corrugation before being refocused by its curvature. This process angularly disperses different wavelengths which in turn are collected by several output waveguides placed on the so-called Rowland circle. By implementing highly reflective DBR gratings in each facet of the diffraction grating and by using optimized waveguide apertures, a low insertion loss can be achieved. More details about PCG design and the working principle can be found elsewhere. ${ }^{16}$ A SEM picture of a PCG (implemented in SOI) that illustrates the working principle is shown in figure $4 .{ }^{16}$ Compared to an AWG, where phase errors can arise due to variations in both the width and height of the arrayed waveguides, a PCG is limited in phase errors due to height variations of a slab waveguide. This can be exploited to design a low crosstalk spectrometer. Still, also the fabrication of the facets should be optimized as corner rounding can be an additional source of phase errors. In the following section we will discuss the use of a PCG for the detection of glucose based on absorption spectroscopy. The choice for a PCG rather than an AWG for this work originates in its potential for low crosstalk.

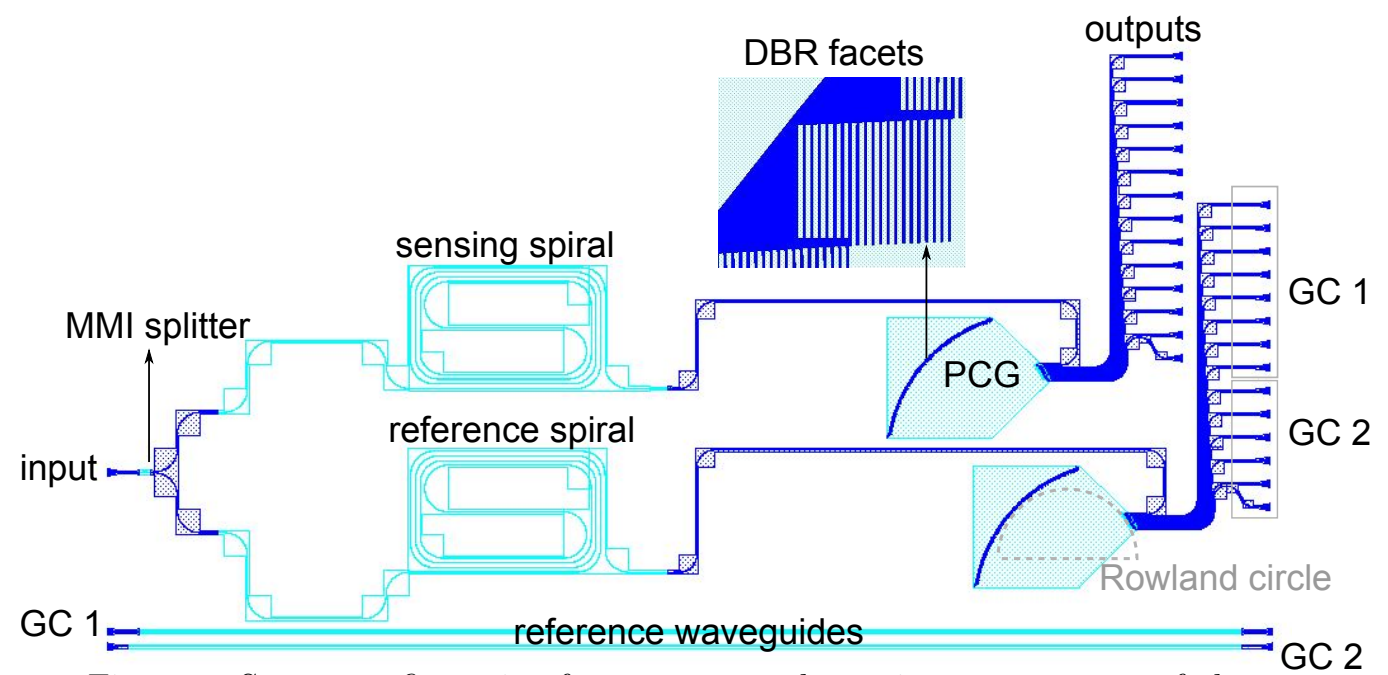

Figure 5: Sensor configuration for evanescent absorption spectroscopy of glucose

Glucose plays a vital role in a variety of biological processes and is especially important for the millions of patients with diabetes. Given the prevalence of sugar related diseases there has been a considerable effort that is still ongoing in the development of a reliable and continuously measuring glucose sensor. ${ }^{17}$ As a part of this effort, we investigate a miniaturized spectrometer, based on Si photonics technology, that can be implanted 
under the skin. In order to succeed for this application, the sensor must be able to continuously and selectively measure glucose in the range of 3 to $20 \mathrm{mM}$ under in vivo conditions for a sufficient duration of time (beyond 6 months). Molecular absorption spectroscopy is the method that we exploit to obtain concentration dependent spectra in the aqueous or serum like environment. Absorption spectroscopy monitors the light attenuation due to the fundamental vibrations or the harmonics of the fundamental vibrations of the glucose molecule. For glucose, two regions of the infrared light spectrum are of special importance: the near-infrared $(0.78-3 \mu \mathrm{m})$ and the mid-infrared $(3-30 \mu \mathrm{m})$. The latter is the traditional region to probe fundamental molecular vibrations which are very strong and can serve as the fingerprint identification of the molecule under investigation. But performing mid-IR spectroscopy using Si photonics technology is challenging as the underlying silica layer starts to heavily absorb wavelengths above $3.5 \mu \mathrm{m}$. As an alternative the first overtone wavelength band (1500-1850 nm) is quite interesting owing to its compatibility with the existing Si photonics technology, but the strength of the signature peaks is 10-20 times weaker than fundamental transitions. This reduced absorption in the first overtone band luckily also holds for the water molecules, the main interferent for in vivo glucose absorption spectroscopy.

To build an on-chip glucose sensor, a high performance spectrometer covering a broad wavelength range with 3-6 $\mathrm{nm}$ resolution is needed. The crosstalk level required for a reliable glucose detection capable of measuring a change of $1 \mathrm{mM}$ concentration is predicted to be at least $30 \mathrm{~dB}{ }^{7}$ To be able to achieve such a good level of crosstalk with standard SOI waveguides is extremely difficult owing to their very high index contrast that induces huge phase errors. This makes it hard to achieve crosstalk level better than $25 \mathrm{~dB}$. The lower index contrast of the $\mathrm{Si}_{3} N_{4}$ platform on the other hand enables crosstalk levels well above $30 \mathrm{~dB}$ as we will show here.

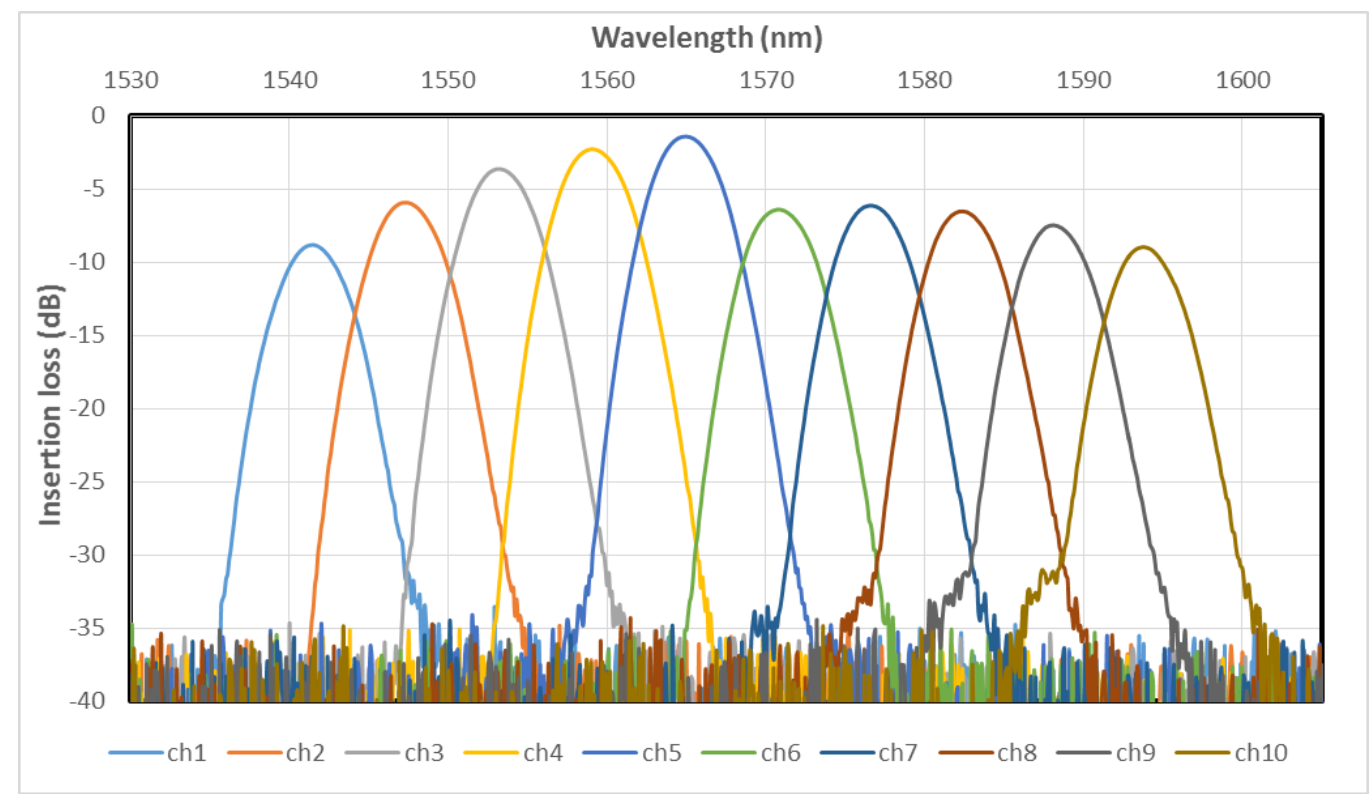

Figure 6: Measured spectral transmission of the planar concave grating device

The sensor design to measure glucose based on absorption spectroscopy is shown in figure 5 . It is a dual-beam configuration with a spiral waveguide for evanescent interaction with aqueous solutions and a planar concave grating (PCG) spectrometer in each arm. This circuit is implemented in $S i_{3} N_{4}$ and it covers one broad glucose absorption feature in the first overtone band. The PCG has a $6 \mathrm{~nm}$ channel spacing and is centered at $1570 \mathrm{~nm}$. The PCG was fabricated on a $300 \mathrm{~nm}$ thick LPCVD silicon nitride stack deposited on top of $3.3 \mu \mathrm{m}$ of underlying oxide on a silicon substrate. The waveguides were formed using $193 \mathrm{~nm}$ optical lithography followed by fluorine based reactive ion etching. Finally the waveguides were cladded with $1 \mu \mathrm{m}$ of top oxide to further lower the loss. High reflectivity grating facets were realized by first order distributed Bragg reflectors (DBRs) that give more than $95 \%$ reflection for the wavelength band of interest. It comprises of 19 periods of fully etched grating 
lines with a period of $500 \mathrm{~nm}$ and a designed fill factor of $43 \%$. For coupling light in and out of the chip grating couplers (GC) were used at the input and output ends. A reference waveguide without the PCG was used to estimate the insertion loss of the device. The result obtained is shown in Fig 6. The nearest neighbor crosstalk and insertion loss at the central channel was measured to be $34 \mathrm{~dB}$ and $1.5 \mathrm{~dB}$ respectively. The reason for the sudden drop in the insertion loss for channel 6 and onwards, is attributed to a different grating coupler used for these channels compared to the input and first five channels. Only one grating coupler response was used to normalize all channels. The roll-off in channel uniformity is attributed to the limited operational bandwidth of the MMI that was used as the $3 \mathrm{~dB}$ splitter. It can be concluded that although the channel uniformity of the complete design needs large improvements, the crosstalk level is low across the whole spectrum. This allows for accurate glucose detection using an integrated spectrometer.

\section{BROADBAND CO-PROPAGATIVE STATIONARY FOURIER TRANSFORM SPECTROMETER}

Fourier transform spectrometers (FTS) are particularly attractive for spectroscopy as they enable broadband operation and provide high spectral resolution. Traditional FTS contain movable components such as the movable mirrors in a Michelson interferometer, which make the spectrometer bulky and prone to mechanical vibrations. To avoid the movable components and to reduce the size of the FTS, several integrated versions have been developed in recent years, including the spatial heterodyne spectrometer (SHS) ${ }^{18}$ and the stationary wave integrated FTS (SWIFTs). ${ }^{19}$ The former requires a large number of waveguide Mach-Zehnder interferometers (MZI) for obtaining a high resolution for a given bandwidth, which indicates a rapidly increasing size of the device. The SWIFTs type, though having high resolution and small footprint, is limited to a small operational bandwidth for a given detector array pitch. The period of the interferogram in the SWIFTs design, given by $\lambda / 2 n_{e f f}$, is usually smaller than the pixel pitch of the detector array that is commercially available. As a result, subsampling is inevitable for the spectral reconstruction which leads to restrictions in the operational wavelength range.

Here we present a novel design of a so-called co-propagative stationary FTS. This design provides a solution for broadband operation in combination with high resolution. In this FTS (fig.7a), an optical signal is first split into two parallel $S i_{3} N_{4}$ ridge waveguides with different ridge width. These two waveguides are properly separated for a desired overlap of the evanescent fields of the two excited waveguide modes. Since the excited modes have a slightly different propagation constant, an interferogram, i.e. the beating pattern of the two waveguide modes, will be generated in between the two waveguides. By placing a fine grating in between the waveguides, we can diffract this interference pattern upwards into a photodiode array. In the following we show how this design effectively lifts the bandwidth restriction of a classical SWIFT design.

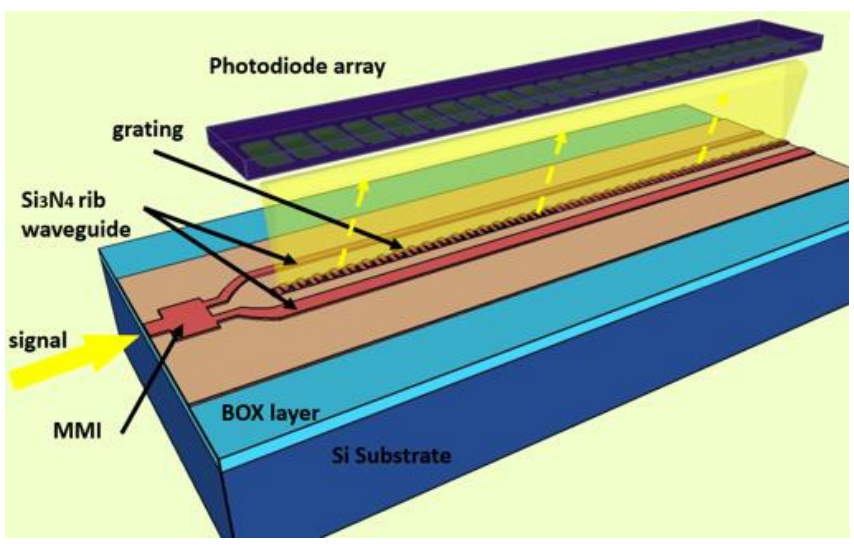

(a)

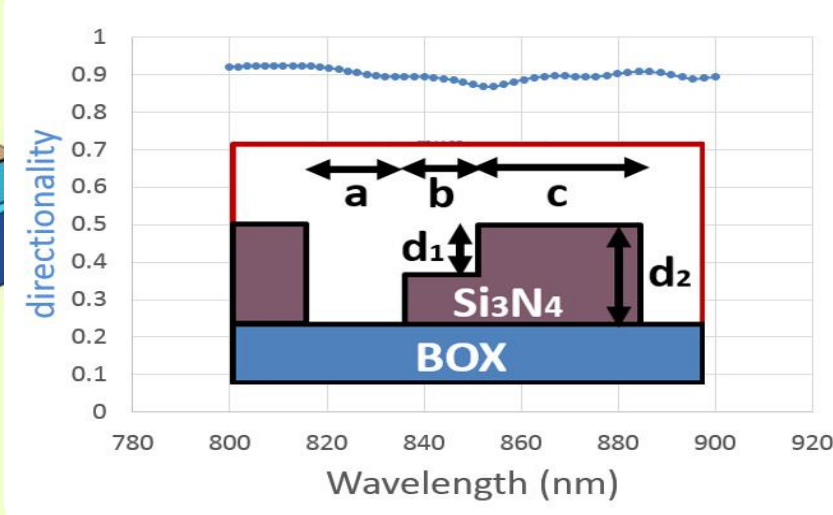

(b)

Figure 7: (a) the sketch of the co-propagative stationary FTS. (b) the side cross section of a grating element, the directionality is calculated for parameter set: $a=180 \mathrm{~nm}, \mathrm{~b}=160 \mathrm{~nm}, \mathrm{c}=280 \mathrm{~nm}, \mathrm{~d}_{1}=150 \mathrm{~nm}$ and $\mathrm{d}_{2}=300 \mathrm{~nm}$. 
Let us consider two waveguide modes characterized by their propagating constant $\beta_{1}$ and $\beta_{2}$ at a certain wavelength $\lambda$. The interference between these two modes has the period of $2 \pi / \Delta \beta$, where $\Delta \beta=\beta_{1}-\beta_{2}=$ $2 \pi \Delta n_{\text {eff }}$ with $\Delta n_{\text {eff }}$ the difference in effective index of the waveguide modes. Hence, compared with SWIFTs, our design provide an increased interferogram period by a factor of $2 n_{\text {eff }} / \Delta n_{\text {eff }}$. According to the sampling theorem of Nyquist-Shannon, subsampling can be avoid as long as the pixel pitch $(\Lambda)$ of the photodiode array is smaller than half of the smallest period of the beatnotes, i.e. $\Lambda<\lambda_{\min } /\left(2 \Delta n_{e f f}\right)$. Consequently, the only limit given by a certain pixel pitch is the lower boundary of the operational bandwidth, which is $2 \Lambda n_{\text {eff }}$. On the other hand, the limit in FTS resolution origins from the finite length $(L)$ of the interferogram. A limited pattern length results in spectral broadening. If we consider a monochromatic input signal, the spectrum will have a $\operatorname{sinc}$-shape, $\sin \left(\pi f_{z} L\right) / \pi f_{z} L$ in Fourier domain, where $f_{z}=\Delta n_{e f f} / \lambda$ in our case. Thus, the attainable spectral resolution is given by the FWHM of this sinc-function, namely $1.207 \lambda^{2} /\left(\Delta n_{e f f} L\right)$.

To give an idea of the performance we can expect, we consider a spectral bandwidth of $100 \mathrm{~nm}$ centered at a wavelength of $850 \mathrm{~nm}$ for the co-propagative stationary FTS. The $S i_{3} N_{4}$ ridge waveguides we use are $300 \mathrm{~nm}$ thick with a shallow etch of $150 \mathrm{~nm}$ and are top oxide cladded. Two ridge waveguides with a width of 300 and $800 \mathrm{~nm}$ provide a difference in effective index of 0.056 (between the fundamental quasi-TE guided modes) at the minimum wavelength of $800 \mathrm{~nm}$. For a propagation of $1 \mathrm{~cm}$, the expected spectral resolution is $1.38 \mathrm{~nm}$ and the period of the interferogram can be calculated as $14.28 \mu \mathrm{m}$, requiring a photodiode array with pixel pitch less than $7.14 \mu \mathrm{m}$ to avoid subsampling, which is already commercially available. Moreover, our device has a small footprint of $0.1 \mathrm{~mm}^{2}$ with a width of approximately of $0.01 \mathrm{~mm}$ and a length of about $10 \mathrm{~mm}$.

In order to measure the inteferogram without losing too much information, the way we design and position the grating is critical. Requirements abound: it should have strong directionality to diffract a large portion of the optical power upwards into the detector; it should ensure weak reflection and get rid of the contradirectional coupling effect as much as possible; ${ }^{20}$ it should have a proper grating strength which, in combination with the optimal gap between both waveguides, ensures that the optical power in the waveguide has a decay of 1/e over the device length for the optimal SNR; and finally, it should be positioned properly for the best contrast of the beating pattern. The last requirement indicates that the evanescent field of the two waveguide modes should have similar strength in the grating region. To be compatible with the $S i_{3} N_{4}$ processing in a $130 \mathrm{~nm}$ node CMOS fab line, we consider the minimum feature size of $150 \mathrm{~nm}$. The asymmetric grating we have chosen for the fabrication of the FTS devices is shown in fig.7b, which has high directionality up to 0.9 in the wavelength range considered. These co-propagative FTS devices are well suited for Raman spectroscopy because of the combination of high bandwidth with small resolution.

\subsection{Conclusion}

Here we have presented the realization of three types of spectrometers (AWG, PCG, FTIR) integrated on a $S_{3} N_{4}$ material platform. This platform is promising for on-chip spectrometers with low insertion loss, low crosstalk and small footprint. These on-chip spectrometers enable a new set of sensors for life-science applications that can be taken outside a lab environment.

\subsection{Acknowledgments}

This work was supported by the FP7-Pocket and the FP7-ERC InSpectra project.

\section{REFERENCES}

[1] Tolstikhin, V., Densmore, A., Logvin, Y., Pimenov, K., Wu, F., and Laframboise, S., "44-channel optical power monitor based on an echelle grating demultiplexer and a waveguide photodetector array monolithically integrated on an InP substrate.," in [Optical Fiber Communication Conference], of America, O. S., ed., Proc. OFC (2003).

[2] Worhoff, K., Heideman, R., Leinse, A., and Hoekman, M., "TriPlex: a versatile dielectric photonic platform," Advanced Optical Technologies , 189-207 (2015).

[3] Ryckeboer, E., Gassenq, A., Muneeb, M., Hattasan, N., Pathak, S., Cerutti, L., Rodriguez, J., Tourni, E., Bogaerts, W., Baets, R., and Roelkens, G., "Silicon-on-insulator spectrometers with integrated GaInAsSb photodiodes for wide-band spectroscopy from 1510 to 2300 nm," Optics Express 21(5), 6101-6108 (2013). 
[4] Gorin, A., Jaouad, A., Grondin, E., Aimez, V., and Charette, P., "Fabrication of silicon nitride waveguides for visible-light using PECVD: a study of the effect of plasma frequency on optical properties.," Optics Express 16(18), 13509-13516 (2008).

[5] Subramanian, A. Z., Neutens, P., Dhakal, A., Jansen, R., Claes, T., Rottenberg, X., Peyskens, F., Selvaraja, S., Helin, P., Bois, B. D., Leyssens, K., Severi, S., Deshpande, P., Baets, R., and Dorpe, P. V., "Low-loss singlemode PECVD silicon nitride photonic wire waveguides for $532900 \mathrm{~nm}$ wavelength window fabricated within a CMOS pilot line.," IEEE Photonics Journal 5(6) (2013).

[6] Kasberger, J., Fromherz, T., Saeed, A., and Jakoby, B., "Miniaturized integrated evanescent field IRabsorption sensor: Design and experimental verification with deteriorated lubrication oil," Vibrational Spectroscopy 56(2), 129-135 (2011).

[7] Ryckeboer, E., "Spectroscopic Detection of Glucose with a Silicon Photonic Integrated Circuit." PhD. Thesis, Ghent University, Belgium (2014).

[8] Neutens, P., Claes, T., Jansen, R., Subramanian, A., Hasan, M. U., Rochus, V., Shirley, F. J., Bois, B. D., Helin, P., Severi, S., Leyssens, K., Dhakal, A., Peyskens, F., Selvaraja, S., Deshpande, P., Baets, R., Lagae, L., Rottenberg, X., and Dorpe, P. V., "Development of a CMOS compatible biophotonics platform based on SiN nanophotonic waveguides.," in [CLEO: QELS Fundamental Science], (2014).

[9] Amatya, R., Holzwarth, C. W., Smith, H., and Ram, R., "Efficient thermal tuning for second-order silicon nitride microring resonators.," IEEE Photonics in Switching 56(2), 149-150 (2007).

[10] Bogaerts, W., Selvaraja, S., Dumon, P., Brouckaert, J., Vos, K. D., Thourhout, D. V., and Baets, R., "Silicon-on-insulator spectral filters fabricated with CMOS technology," IEEE Journal of Selected Topics in Quantum electronics 16, 33-44 (2010).

[11] "The European FP7 Pocket project." https://http://www.pocket-proj.eu/. (Accessed: 1 March 2016).

[12] Smit, M. and Dam, C. V., "PHASAR-based WDM-Devices: principles, design and applications," IEEE Journal of Selected Topics in Quantum electronics 2, 236-250 (1996).

[13] Martens, D., Subramanian, A., Pathak, S., Vanslembrouck, M., Bienstman, P., Bogaerts, W., and Baets, R., "Compact silicon nitride arrayed waveguide gratings for very near-infrared wavelengths," Photonics Technology Letters 27, 137-140 (2015).

[14] Martens, D., Stassen, A., Roy, W. V., and Bienstman, P., "Novelty low-cost integrated photonic biosensor using broadband source and on-chip spectral filter," in [Proceedings Symposium IEEE Photonics Society Benelux], 173-176 (2015).

[15] Wang, X. and Madsen, C. K., "Highly sensitive compact refractive index sensor based on phase-shifted sidewall Bragg gratings in slot waveguide.," Applied optics 53(1), 96-103 (2014).

[16] Brouckaert, J., "Integration of Photodetectors on Silicon Photonic Integrated Circuits (PICs) for Spectroscopic Applications." PhD. Thesis, Ghent University, Belgium (2010).

[17] Smith, J., [The Pursuit of Noninvasive Glucose: Hunting the Deceitful Turkey], Mendosa (2015). fourth edition.

[18] Florjanczyk, M., Cheben, P., Janz, S., Scott, A., Solheim, B., and Xu, D., "Planar waveguide spatial heterodyne spectrometer," Photonics North 2007 (2007).

[19] le Coarer, E., Blaize, S., Benech, P., Stefanon, I., Morand, A., Lrondel, G., Leblond, G., Kern, P., Fedeli, J. M., and Royer, P., "Wavelength-scale stationary-wave integrated Fourier-transform spectrometry.," Nature Photonics 1(8), 473-478 (2007).

[20] Shi, W., Wang, X., Lin, C., Yun, H., Liu, Y., Baehr-Jones, T., Hochberg, M., Jaeger, N. A. F., and Chrostowski, L., "Silicon photonic grating-assisted, contra-directional couplers.," Optics Express 21(3), 3633-3650 (2013). 